\title{
DSM-based Knowledge Transfer Modeling Between Projects for Multi-project Clustering Analysis
}

\author{
Yingxin $\mathrm{Bi}^{1}$, Qing Yang*1, Mingxing Chang ${ }^{1}$, Tao $\mathrm{Yao}^{2}$ \\ ${ }^{1}$ School of Economics and Management, University of Science \& Technology Beijing, China \\ ${ }^{2}$ Alibaba US, 500 108th Ave NE \#800, Bellevue, WA 98004, USA
}

\begin{abstract}
Overlapping is a typical feature of concurrent multi-project, while it is challenging that building interdependency relationships among all the projects in the program, especially from the view of knowledge dependency. However, knowledge transfer needs to be considered to improve concurrent multi-project management. Sharing knowledge among projects to improve the performance of multi-project is one of the important characteristics of project management. Hence, we propose a model to measure the connection strength among all the projects based on knowledge transfer. Further, taking the results of design structure matrix (DSM) as input, we suggest a two-stage clustering criterion to make projects with secure knowledge connection strength form a program. Finally, an example is provided to verify the effectiveness of the proposed model. Through the clustering of projects, it is more conducive to improving knowledge transfer among projects in the cluster, so as to shorten the time of multiple projects.
\end{abstract}

Keywords: multi-project management; knowledge transfer; design structure matrix; connection strength; clustering analysis

\section{Introduction}

Organizational learning and knowledge transfer are the key to enhance the competitiveness of enterprises (Egelman et al., 2016). However, learning and knowledge transfer are rarely or never mentioned in program management.

It is not possible for program management, such as aligning, plan coordination and implementation of several attached projects, to achieve benefits if these projects are managed separately. The most significant difference between program and project management exists in coherence and efficiency of relationships within the projects. However, it can't insight any program where the projects are irrelevant in any sense, and it is challenging as well that building interdependency relationships among all the projects in the program (Vukomanovć, 2016).

Egelman et al. (2016) highlighted that overlapping of product generations can improve productivity significantly. Hence, this paper will focus on the research of the overlapping relationship between concurrent projects, to explore the connection strength among projects, by the method of clustering to improve the efficiency of multi-project. With the clustering of projects, it is more conducive to alter the knowledge transfer within projects and shorten the duration of multiple projects (program) for reducing total costs (Yang et al., 2015). 
Consequently, this paper proposes the model of the connection strength of concurrent multi-project based on knowledge transfer. Furthermore, we propose a two-stage clustering criterion to make projects with strong knowledge connections form a program.

\section{Literature Review}

This research observes that knowledge transfer (KT) plays a vital role in multi-project management.

Özkan-Seely et al. (2015) state that KT from the product to the progress design team transfer information such as customer sections and coveted goods specifications. According to Egelman et al. (2016), having more generations of the focus product is profitable to organization productivity above and beyond the benefits of traditional KT. Yang et al. (2014) measured interaction strength among teams based on design structure matrix(DSM) properly, as shown in Figure 1, and highlighted that the clustered units can reduce coordination time significantly. The clustering of models in organizations with related property is primary to verify a more effective production process and assets portion in mass customized production systems (Anzanello and Fogliatto, 2011).

KT between teams may be constant, front-loaded, back-loaded, U-shaped (Özkan-Seely et al., 2015). Egelman et al. (2016) find that productivity modifies when multiple generations of the same goods are produced concurrently. Vuorinen and Martinsuo (2018) explain the investigation of the integration execution uncovered the existing utility of several personal and group integration carry out in the changeable environment. The later the knowledge is sent, the higher the knowledge maturity will be. The more reliable the ability to learn, reflecting the greater shortening of time (Rauniar et al., 2019), as the Figure 2 shows.

Therefore, in this paper, we use DSM to build multi-project dependency from the perspective of knowledge transfer and acceptance. In order to demonstrate the significance of information sharing and knowledge learning, after the proposed clustering method, the projects with strong knowledge interdependency form a program.

\section{The Model Formulation}

\subsection{Building the Design Structure Matrix (DSM) of Knowledge Transfer}

The DSM is used to represent the time factor matrix of knowledge transfer and acceptance so as to quantify the impact of overlapping on knowledge interaction between projects. Matrix $O$, as shown in Figure 1(a), indicating that the transfer time factor $O$ is the percentage of the time that the project transfers knowledge of its total time, $t_{B} / d_{B}$. Matrix $I$, as shown in Figure 1(b), indicating that the acceptance time factor $I$ represents the percentage of the time that the project accepts knowledge and information, $t_{C} / d_{C}$. The number of the lower diagonal and the upper diagonal mean the feed forward overlapping and the feedback overlapping, respectively.

For example, $O(2,1)=0.6$ in Figure 1(a) shows that when project A corresponding to the first column reaches $60 \%$ of its time, it begins to transfer knowledge to project $\mathrm{B}$ 
corresponding to the second row. $I(2,1)=0.2$ in Figure $1(\mathrm{~b})$ shows that project B starts to receive information from project $\mathrm{A}$ at $20 \%$ of its time.

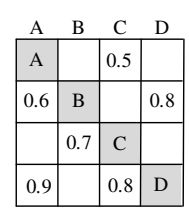

(a) $O$ matrix

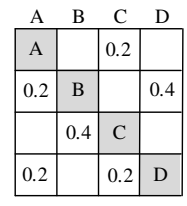

(b) I matrix

Figure 1. DSM describing knowledge transfer and acceptance between projects

\subsection{Measuring Connection Strength of Concurrent Multi-project based on Knowledge Interaction}

As shown in Figure 2, for the project sending the knowledge, its knowledge maturity function is an increasing function over time, the maximum value will eventually be reached. For the project receiving the knowledge, the sensitivity function of organizational learning decreases over time as it accepts earlier knowledge. The more beneficial the information is to an organization's learning, the learning ability increases, reflecting a shortened duration for the project. In this paper, we define the knowledge transfer and acceptance as knowledge interaction.

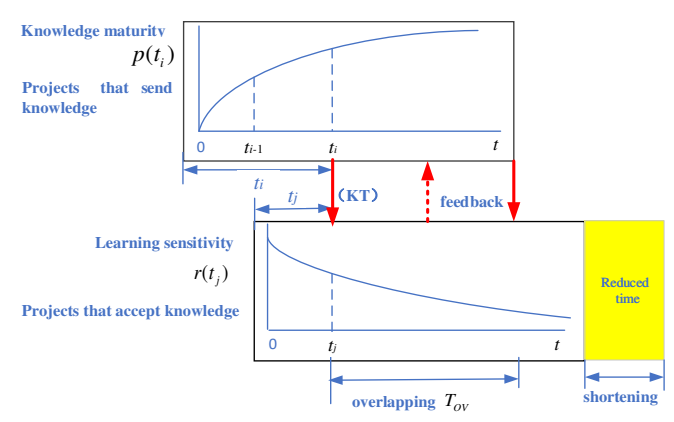

Figure 2. Knowledge maturity and learning sensitivity related to project overlapping

3.2.1 Modeling knowledge transfer capability of sending knowledge project in the concurrent process

Özkan-Seely et al. (2015) proposes that knowledge development (KD) is related to the changing rate of $\mathrm{KD}(\gamma(t))$, the team's current knowledge level $(\mathrm{D}(t))$ and the diminishing rate of return $\left(\rho_{1}\right)$, which means that the KD function at time $\mathrm{t}$ is $\gamma(t)[\mathrm{D}(t)]^{\rho_{1}}$. Therefore, when project $i$ initially transfers knowledge to $j$, according to the knowledge maturity function (see Fig. 2), the accumulated amount of knowledge of project $i$ is:

$$
K M(i, j)=\int_{0}^{O(i, j)} \gamma[O(i)+1]^{\rho} d_{O(i)}=\gamma \frac{1}{\rho+1}[O(i, j)+1]^{\rho+1}
$$


where $\mathrm{D}(i)$ represents the duration of project $i$ and $\rho \in(0,1)$ is the diminishing rate of return (Özkan-Seely et al., 2015). In this paper, $\gamma\left(t_{i}\right)$ is set as a constant and $\rho=0.9, \gamma$ $=0.5$.

3.2.2 Modeling knowledge absorptive capability of receiving knowledge project in the concurrent process

By knowledge transfer and the acceptance of the DSM, the overlapping time between $i$ and $j$ can be calculated:

$$
T_{O V}(i, j)=\left\{\begin{array}{cc}
(1-I(i, j)) \times D(i) & \text { if }(1-I(i, j)) \times D(i)<(1-O(i, j)) \times D(j) \\
(1-O(i, j)) \times D(j) & \text { else }
\end{array}\right.
$$

Therefore, the ratio of time overlapping between the two projects is:

$$
\operatorname{ROV}(i, j)=T_{O V}(i, j) /(D(i)+D(j))
$$

For the project $i$ and $j$, the learning sensitivity function of the project $j$ receiving knowledge using the DSM matrix is:

$$
L S(i, j)=\exp \left(g\left(t_{j}\right)(1-I(i, j) \times R O V(i, j))-1\right.
$$

where $(1-I(i, j))$ represents the percentage of the work remaining when the project accepts the knowledge, $g\left(t_{j}\right)$ represents the inherent ability of the receiving information team to digest and absorb knowledge, which may change over time. In this paper, we define $g$ as a constant $(g=1.5)$.

\subsubsection{Connection strength of concurrent multi-project}

The connection strength between project $i$ and project $j$ due to knowledge transfer is related to the following factors: the accumulated knowledge $K M(i, j)$ of project $i$ when sending the initial knowledge; the learning sensitivity $L S(i, j)$ when accepting the knowledge of project $j$; and the project communication in the parallel process, which is related to the amount of overlapping.

Furthermore, the connection strength based on knowledge transfer $\operatorname{CSKT}(i, j)$ related to the learning ability between the two projects can be obtained, which is the knowledge maturity of the sending project (KM) and the knowledge absorption sensitivity of the receiving project (LS):

$$
\operatorname{CSKT}(i, j)=K M(i, j) \times L S(i, j)
$$

\subsection{Two-stage Clustering Criterion of Multi-project}

The purpose of DSM clustering is generally to maximize the interaction between elements within the cluster and minimize the interaction between clusters. The clustering criterion 
proposed in this paper is to maximize the connection strength, between projects within the cluster, related to knowledge interaction, and to maximize the total time (intra-project and inter-project) shortened by learning. Therefore, this paper adopts two-stage clustering method.

\subsubsection{The First-stage clustering criterion}

Firstly, the purpose of the clustering criterion is that the connection strength related to knowledge interaction is maximized internally within the cluster. It refers to the ratio of the weighted sum of the connection strength of the selected project in one cluster with other projects (that is, the weighted sum of non-zero elements in the corresponding rows and columns of the project in the cluster) to the total connection strength of the project in the entire organization with other projects (that is, the sum of non-zero elements in the rows and columns of the project in the whole DSM) (see Fig. 3).

\begin{tabular}{|c|c|c|c|c|c|c|c|c|}
\hline & $A$ & $B$ & \begin{tabular}{l|l}
$C$ \\
\end{tabular} & $D$ & $E$ & $F$ & $G$ & \multirow{3}{*}{$\begin{array}{l}\text { Added connection strength } \\
\text { (row-accepted and column- }\end{array}$} \\
\hline$A$ & A & & & & & & & \\
\hline$B$ & & $B$ & & $x$ & $x$ & & & \\
\hline C & $x$ & $x$ & $C$ & $x$ & $x$ & & & \\
\hline$D$ & & $x$ & $x$ & $D$ & $x$ & & & The selected project \\
\hline$E$ & & & $x^{2}$ & $x$ & E & $x$ & & \\
\hline$F$ & $\times$ & & & & $x$ & $F$ & $x$ & Connection strength \\
\hline$G$ & & & & $x$ & & $x$ & $G$ & \\
\hline
\end{tabular}

Figure 3. Example of the added interaction strength

Hence, we build Added Internal and External Connection Strength Ration (AIECR), as shown in Fig. 3, the first-stage clustering criterion is to maximize AIECR, in this way, it can make the connection strength between projects within the cluster stronger, that is, projects with strong knowledge interaction are in one cluster. It can be calculated with equation (14):

$$
\max \operatorname{AIECR}\left(\text { cluster }_{k}\right)=\frac{\left(\omega_{1} \times \sum_{i=n_{k}}^{m_{k}-1} \operatorname{CSKT}\left(i, m_{k}\right)+\omega_{2} \times \sum_{j=n_{k}}^{m_{k}-1} \operatorname{CSKT}\left(m_{k}, j\right)\right)^{\alpha}}{\omega_{1} \times \sum_{i=1}^{N} \operatorname{CSKT}\left(i, m_{k}\right)+\omega_{2} \times \sum_{j=1}^{N} \operatorname{CSKT}\left(m_{k}, j\right)}
$$

where $k$ is the cluster of the selected project during each iteration, $n_{k}$ and $m_{k}$ are the first element and last element in cluster $k$ respectively. $\sum_{i=n_{k}}^{m_{k}-1} \operatorname{CSKT}\left(i, m_{k}\right)+\sum_{j=n_{k}}^{m_{k}-1} \operatorname{CSKT}\left(m_{k}, j\right)$ is the sum of added connection strength of the selected project with other projects in cluster $k$. $\alpha$ is a penalty coefficient. $N$ represents the total number of projects, where $\omega_{1}, \omega_{2}$ are weight coefficients and $\omega_{1}+\omega_{2}=1$.

Note that $A I E C R\left(\right.$ cluster $\left._{k}\right)$ is calculated randomly selected project in the clustering algorithm. The role of AIECR( cluster $_{k}$ ) in the algorithm is like a bid (Thebeau, 2001). Any project in DSM has the same probability of being selected. Once a project is chosen, the algorithm calculates a bid from each cluster. Compared with the traditional clustering 
goal of maximizing the AIS ( cluster $_{k}$ ) (Yang et al., 2014), AIECR( cluster $_{k}$ ) can avoid the problem of small cluster size after clustering.

\subsubsection{The second-stage clustering criterion}

The second stage clustering criterion is to minimize the total transfer knowledge time, including both internal and external transfer knowledge time. The Internal Knowledge Transfer Time (IKT) refers to communicating knowledge inside a cluster and depends on the knowledge transfer frequency $K T F\left(c l_{k}\right) . K T F\left(c l_{k}\right)$ and $K T F($ cell_out $)$ can be captured by equation (7):

$$
K T F\left(c l_{k}\right)=\mu_{0} \exp \left\{-\frac{1}{\left(\max \left(\left(c l_{k}-\left(c l_{0}-1\right)\right), 1\right)\right)^{\eta}}\right\}
$$

where $\mu_{0}$ represents the uncertainty of information, $c l_{0}$ is the reasonable size of a cluster, $\eta$ is a penalty coefficient.

Similar to IKT, the External Knowledge Transfer Time (EKT) refers to the transfer time among projects outside the cluster and determined by $K T F($ cell_out $)$. It can be calculated with equation (8):

$$
K T F(\text { cell_out })=\mu_{0} \exp \left\{-\frac{1}{\left(\max \left(\left(\text { cell_out }-\left(\text { cell_out } t_{0}-1\right)\right), 1\right)\right)^{\lambda}}\right\}
$$

where $c e l l \_$out is the total number of projects outside the cluster, cell_out $t_{0}$ is the ideal number of projects outside the cluster, $\lambda$ is a penalty coefficient.

Therefore, the Internal Knowledge Transfer time (IKT) and External Knowledge Transfer Time (EKT) can be calculated as equation (9) and (10), respectively.

$$
I K T=\sum_{k=1}^{N_{C}}\left(\left(\sum_{i=n_{k}}^{m_{k}} \sum_{j=n_{k}}^{m_{k}} \operatorname{CSKT}(i, j)\right) \times K \operatorname{KTF}\left(c l_{k}\right)\right) \quad i \neq j
$$

where $N_{C}$ is the number of clusters in the DSM.

$$
E K T=\sum_{k=1}^{N_{C}}\left(\left(\sum_{i=n_{k}}^{m_{k}}\left(\sum_{j=1}^{n_{k}-1} C S K T(i, j)+\sum_{j=m_{k}+1}^{N} C S K T(i, j)\right) \times K T F(\text { cell_out })\right)\right)
$$

Based on the results above, the second-stage clustering objective is minimizing the weighted total transfer knowledge time including both IKT and EKT.

$$
\text { Min: } \quad T K T=v_{1} I K T+v_{2} E K T
$$

Subject to: $\quad c l_{k} \leq c l_{0}$ 
where $v_{1}, v_{2}$ are weight coefficients and $v_{1}+v_{2}=1$. In order to avoid too many elements, we take $v_{1}<v_{2}$.

\section{Case Study}

An illustrative example, F company optical communication product R\&D projects (FOC), including current version and new version. The 10G EPON strategic multi-project set consists of 14 projects, covering three levels of OLT / ONU optical communication transceiver, lasers, and chips. For example, at the same time of chip R\&D, the laser and the optical communication transceiver should utilize the external chip with the same index to conduct synchronous $\mathrm{R} \& \mathrm{D}$, which can improve the degree of concurrent process and shorten the R\&D cycle. Based on the responses and other information provided, we built the $O$ matrix and $I$ matrix of (see Fig. 4). In this case, the knowledge between projects cannot learn from each other. For example, as project B and C, when project B corresponding to the first column reaches $40 \%$ of its time, it begins to transfer knowledge to project $\mathrm{C}$ corresponding to the second row. At the same time, project $\mathrm{C}$ starts to receive information from project $\mathrm{B}$ at $30 \%$ of its time. While the project $\mathrm{C}$ cannot learn from project B. The value of factor $O$ and $I$ influence the connection strength among projects. In equations (6)-(11), $\omega_{1}=0.1, \omega_{2}=0.9, \alpha=4, \mu_{0}=2.5, \eta=1, \lambda=1, v_{1}=0.1, v_{2}=0.9$, which are empirical values according to the project manager's knowledge and experience.
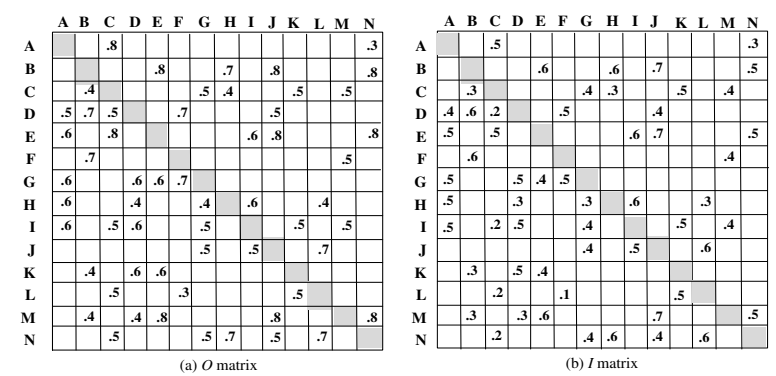

Figure 4. FOC projects DSM describing knowledge transfer and acceptance

We calculated the connection strength with equations (1)-(9) and the shorten time between projects with equations (10)-(13) and applied the two-stage clustering procedure in the Matlab® 15 software. The normalized initial 14 FOC projects DSM can be obtained as shown in Figure 5, the size of the dots indicates the degree of connection strength, and the clustering result is shown in Figure 5. 

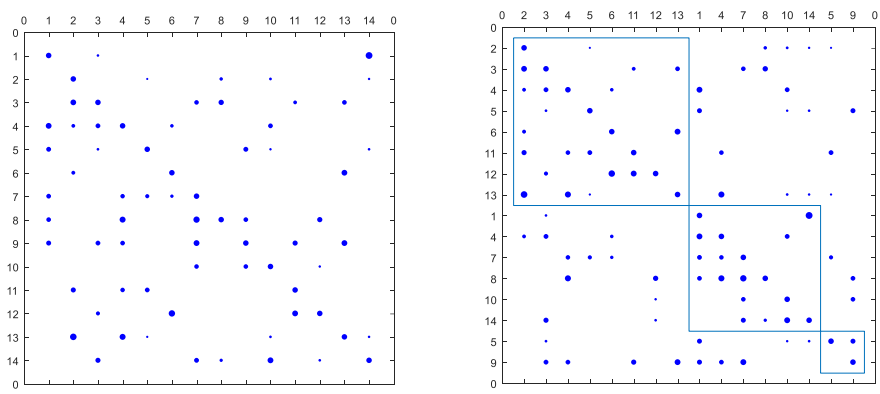

Figure 5. Initial and clustered connection strength matrix between projects

It can be seen that the larger value of the project's knowledge transfer time factor $O$ and the smaller value of the project's knowledge acceptance time factor $I$, the greater the connection strength based knowledge among projects, which indicates that the learning ability among projects is stronger. What's more, after clustering, the 14 FOC projects are divided into three working groups, and the projects with strong knowledge transfer and learning are gathered into one working group, making the projects within the cluster easier to communicate, so as to reduce the time of the multi-project. So, they are more important in the multi-project management with clustering based on knowledge transfer and learning ability.

\section{Conclusion}

In this paper, we build interdependency relationships among all the projects from the perspective of knowledge transfer to enhance multi-project management. Firstly, this paper proposes the model of the connection strength of concurrent multi-project based on knowledge transfer, and then in order to make projects with strong knowledge connection strength form a program, we suggest a two-stage clustering criterion.

The main limitations of this research are: (1) we encourage future studies to consider the factor of the knowledge transfer cost to improve the second-stage clustering method; (2) for further analysis, the part of learning curve should consider to measure the shorten time of the project; (3) we should consider the factor of knowledge feedback in the further study, namely, the projects can learn from each other.

\section{Acknowledgments}

This study was supported by the National Natural Science Foundation of China (No.71872011 and No.71929101) and Fundamental Research Funds for the Central Universities (FRF-BR-18-001B). 


\section{References}

Anzanello, M. J., Fogliatto, F. S., 2011. Selecting the best clustering variables for grouping masscustomized products involving workers' learning. International Journal of Production Economics, 130(2), 268-276.

Egelman, C. D., Epple, D., Argote, L., Fuchs, E. R. H., 2016. Learning by Doing in Multiproduct Manufacturing: Variety, Customizations, and Overlapping Product Generations. Management Science, 63(2), 405-423.

Vukomanovć, M., Young, M., Huynink, S., 2016. IPMA ICB 4.0 - A global standard for project, programme and portfolio management competences. International Journal of Project Management, 34(8):1703-1705.

Özkan-Seely, G. F., Gaimon, C., Kavadias, S., 2015. Dynamic Knowledge Transfer and Knowledge Development for Product and Process Design Teams. Manufacturing \& Service Operations Management, 17(2), 177-190.

Rauniar, R., Rawski, G., Morgan, S., 2019. Knowledge integration in IPPD project: role of shared project mission, mutual trust, and mutual influence. International Journal of Project Management, 37(2):239-258.

Thebeau, R., 2001. Knowledge management of system interfaces and interactions for product development processes. Master's thesis, MIT Press, Cambridge, MA.

Vuorinen, L., Martinsuo, M., 2018. Program integration in multi-project change programs: agency in integration practice. International Journal of Project Management, 36(4), 583-599.

Yang, Q., Yao, T., Lu, T., Zhang, B., 2014. An overlapping-based design structure matrix for measuring interaction strength and clustering analysis in product development project. IEEE Transactions on Engineering Management, 61(1):159-170.

Yang, Q., Yang, N., Browning, T. R., Jiang, B., Yao, T., 2020. Clustering product development project organization from the perspective of social network analysis. IEEE Transactions on Engineering Management, DOI: 10.1109/TEM.2019.2939398.

Yang, Q., Kherbachi, S., Hong, Y. S., Shan, C., 2015. Identifying and managing coordination complexity in global product development project. International Journal of Project Management, 33(7), 1464-1475.

Contact: Qing Yang, University of Science \& Technology Beijing, School of Economics and Management, No.30 Xueyuan Road, Haidian District, Beijing 100083, China, 86+13911463353, yangqing@manage.ustb.edu.cn. http://dsem.ustb.edu.cn/jgen/a/Faculfy_List/20141009/124.html 